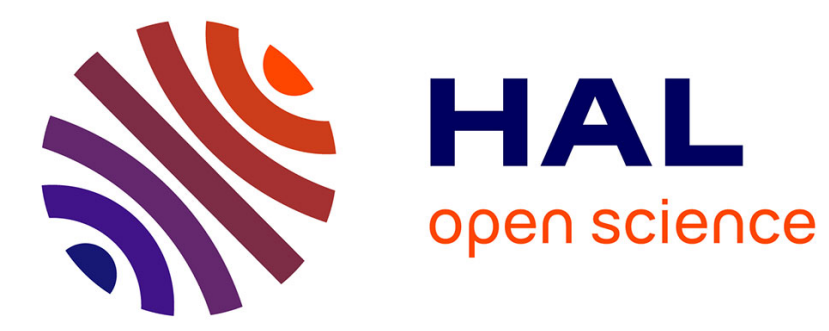

\title{
First results of Wavefront sensing on SOTA
}

Nicolas Védrenne, Marie-Thérèse Velluet, Cyril Petit, Vincent Michau, Julien Chabé, Aziz Ziad, Duy-Ha Phung, Nicolas Maurice, Etienne Samain, Géraldine Artaud, et al.

\section{- To cite this version:}

Nicolas Védrenne, Marie-Thérèse Velluet, Cyril Petit, Vincent Michau, Julien Chabé, et al.. First results of Wavefront sensing on SOTA. 2015 IEEE International Conference on Space Optical Systems and Applications (ICSOS), pp.1-8, 2015, 10.1109/ICSOS.2015.7425087 . hal-01429768

\section{HAL Id: hal-01429768 \\ https://hal.science/hal-01429768}

Submitted on 12 Nov 2021

HAL is a multi-disciplinary open access archive for the deposit and dissemination of scientific research documents, whether they are published or not. The documents may come from teaching and research institutions in France or abroad, or from public or private research centers.
L'archive ouverte pluridisciplinaire HAL, est destinée au dépôt et à la diffusion de documents scientifiques de niveau recherche, publiés ou non, émanant des établissements d'enseignement et de recherche français ou étrangers, des laboratoires publics ou privés.

\section{다)(1) $(5$}

Distributed under a Creative Commons Attribution - NonCommercial| 4.0 International 


\title{
First results of Wavefront sensing on SOTA
}

\author{
Nicolas Védrenne, Marie-Thérèse Velluet, Cyril Petit, \\ Vincent Michau \\ Theoretical and Applied Optics Department \\ ONERA, The French Aerospace Lab \\ 92322 Châtillon Cedex, France \\ Nicolas.vedrenne@,onera.fr
Duy-Ha Phung, Nicolas Maurice, Etienne Samain OCA-Géoazur
2130, Route de l'Observatoire, 06460 Saint-Vallier-de-Thiey, France

\author{
J. Chabé, A. Ziad \\ Lagrange - UMR7293 \\ Parc Valrose \\ 06108 Nice Cédex 02, France
}
Géraldine Artaud, Jean-Luc Issler DCT/RF/ITP
CNES
18 , av. Edouard Belin,

31401 Toulouse Cedex 9, France

\author{
Morio Toyoshima, Maki Akioka, Dimitar Kolev, Yasushi Munemasa, Hideki Takenaka, Naohiko Iwakiri \\ NICT \\ 4-2-1 Nukui-kita, \\ Koganei, Tokyo, 184-8795 Japan
}

\begin{abstract}
For satellite to ground laser links, atmospheric turbulence is a major cause of impairments. The induced phase perturbations along the propagation path cause beam scintillation in the receiver plane and they can also severely compromise the coupling of the flux into a receiver of limited size. To address these impairments, dedicated mitigation strategies must be developed. This requires accurate understanding of the perturbation origin. Beam propagation models have demonstrated their ability to reproduce statistical characteristics of optical perturbations on a satellite to ground laser link for elevations as low as $\mathbf{2 0}^{\circ}$. For smaller elevations, measurements performed on stars illustrated the limits of analytical approaches and the interest for end-to-end models. We report here the first propagation channel measurements performed on a LEO microsatellite with a Shack-Hartmann wavefront sensor (WFS). The laser beam at $976 \mathrm{~nm}$ provided by SOTA optical terminal have been analyzed with a ShackHartmann wavefront sensor located at Coudé focus of the French ground station (1,55 m MéO telescope) in July 2015. Wavefront characteristics and scintillation patterns recorded with the WFS are analyzed and compared to atmospheric turbulence perturbations model fed with in situ measurements of atmospheric parameters retrieved from GDIMM.
\end{abstract}

Keywords-Wavefront sensing; optical turbulence; adaptive optics;free space laser communications

\section{INTRODUCTION}

High data rate optical communications from satellite (LEO or GEO) to ground have been under investigation for several years; they offer a good alternative to the RF links which get to the limit of their capabilities. However, one of the major issues is the atmospheric turbulence which disrupts the propagation channel. Both phase and amplitude of the optical wave are perturbed leading to amplitude fluctuations of the signal and potential fadings. Mitigation techniques can be applied on the signal itself (interleaving or corrector error codes) and/or on the wavefront (adaptive optics systems). To optimize these techniques, propagation channel characterization is crucial, in particular the temporal and spatial statistics of wave perturbations. Dedicated numerical models have been developed [1][2] and validated on stars but never on a real optical link emitted from a LEO satellite.

We report here wavefront measurements performed with a laser link established between the MéO telescope, an experimental ground station belonging to the Observatoire de la Côte d'Azur (OCA) (Calern, France) and the SOTA terminal of the SOCRATES micro-satellite [3]. The experiment performed with SOTA is part of the framework cooperation agreement signed between NICT and CNES on information and communication technology. The optical ground station is developed and managed through a project called DOMINO (Demonstrator for direct Optical transMission at hIgh data rate iN Orbit). CNES is the project owner. The Côte d'Azur observatory is in charge of the whole experiment, conducting the studies, development and the measurement campaign with the support of ONERA for the turbulence characterization and adaptive optics compensation. Airbus Defense and Space for system analysis and Thales Alenia Space for communication aspects.

The first part of the paper presents the experimental setup and parameters. Second part describes the data collected. They are analyzed in the third section and compared with numerical models. Some discussion allows to conclude on the channel characterization and to give recommendations for considering turbulence along the line of sight. 


\section{EXPERIMENTAL SETUP}

\section{A. SOTA terminal and ground station}

The Small Optical TrAnsponder (SOTA) terminal is a technical demonstrator of embedded laser communication technologies on small satellites [3]. SOTA provides two possible downlink wavelengths: $1549 \mathrm{~nm}$ and $976 \mathrm{~nm}$. The future optical space transmissions operational systems to which CNES might be involved in will use 1.55 micron wavelength range for uplink and downlink, according to aimed future CCSDS standards under development [4], compatible with the LEO-direct-to-Earth transmission scenarii. For this experiment, the $976 \mathrm{~nm}$ modulated laser of $200 \mathrm{~mW}$ average power was used in order to benefit from the existing ODISSEE optical bench available at OCA. The light emitted by the terminal onboard the satellite is collected by the $1.5 \mathrm{~m}$ telescope of the French Optical Ground Station $\mathrm{MeO}$ [5] located in the hinterland of Grasse at 1270 meters above sea level.

\section{B. Wavefront sensor of ODISSEE}

Wavefront analysis is performed using an adaptive optics (AO) bench located at the Coude focus of the ground station. The ODISSEE AO optical bench was designed for investigation of various applications, including visible imaging. The wavefront sensing was performed both in open loop and in closed loop. The AO bench is illustrated in Fig. 1. The light from Coudé focus enters the bench at the top-left of the image. The field rotation is compensated by a pupil derotator. The pupil position is stabilized with a fast tip/tilt mirror located close to a focal plane. A second tip/tilt mirror is used to compensate for atmospheric turbulent tip/tilt, pointing residuals and vibrations. After reflecting on the deformable mirror $(88$ actuators Cilas stack array mirror [7]), the beam is directed onto the WFS.

The wavefront sensor (top right of Fig. 1) is a custom Shack Hartmann WFS with $8 \times 8$ square subapertures [2]. The size of a subaperture is $19 \mathrm{~cm}$ in the entrance pupil plane of the system. The focal plane of each subaperture is sampled with 30 x 30 pixels. The focal length of the microlenses has been chosen so that Shannon sampling factor is 0.5 at $600 \mathrm{~nm}$ (one diffraction spot per pixel). The WFS camera is a First Light Imaging $^{\mathrm{TM}} \mathrm{OCAM}^{2}$ camera including a deep depletion E2V chip of $240 \times 240$ pixels providing 0.3 quantum efficiency at $970 \mathrm{~nm}$. Slopes and intensities per subaperture are recorded with a frame rate of $1450 \mathrm{~Hz}$. The slope computation algorithm is a thresholded Center of Gravity. The threshold value is adjusted to limit background noise influence on the slope measurements. The total intensity in each sub-aperture is computed by the addition of the pixel thresholded values.

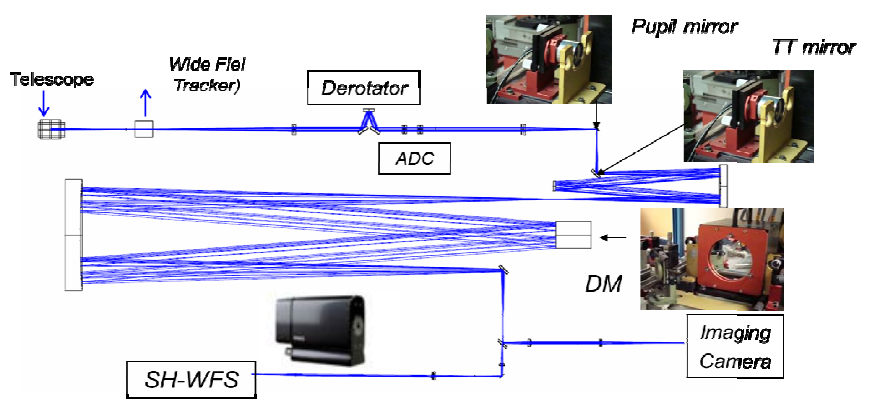

Fig. 1. Adaptive optics bench at Coudé focus of the MéO ground station.

\section{GDIMM}

During acquisitions with SOTA a Generalized Differential Image Motion Monitor (GDIMM) [6] was monitoring parameters of the optical turbulence (Fried parameter, averaged scintillation index on the instrument aperture and isoplanatic angle) on a bright star (Vega). GDIMM is based on the use of a small telescope equipped with a 3-holes mask at its entrance pupil. The Fried parameter is retrieved from differential slopes measurements as measured by the classical DIMM technique using two sub-pupils of the mask $(6 \mathrm{~cm}$ diameter separated by a distance of $20 \mathrm{~cm}$ ). The isoplanatic angle is estimated from scintillation through the third subpupil (its diameter is $10 \mathrm{~cm}$, with a central obstruction of 4 $\mathrm{cm})$. GDIMM was located at $80 \mathrm{~m}$ South-West of the telescope.

\section{MEASUREMENTS}

\section{A. Data acquired}

The WFS data were collected on the $21^{\text {st }}$ of July. The satellite rises at 22:34:13 s UTC. At 22:34:58, 200 raw images from the WFS were acquired (elevation $7.5^{\circ}$ ). Between 22:35:40 and 22:35:55 the parameters of the WFS (camera gain, threshold level, and background) were adjusted. Data acquisition (slopes and intensities) was launched at 22:36:09 (elevation $13^{\circ}$ ). At 22:36:56 (elevation $21^{\circ}$ ) AO loop was closed and remained closed until 22:38:41 (44.5 $)$. Between 22:36:09 and 22:37:30, a few parameters like the gain of the camera and the threshold level were adjusted. Pupil stabilization was also launched during this period. During the first part of the pass, the various parameter adjustments impact the measurement of the received flux. The measurements of flux fluctuation (scintillation index) are altered. The phase measurements are disrupted by the closing of the loop and resumed when the loop is stabilized. From the beginning of the acquisition to the end, $152 \mathrm{~s}$ of slopes and intensities were gathered at $1450 \mathrm{~Hz}$. The parameters of the line of sight (LOS) and main events of the acquisitions are illustrated in Fig. 2. 

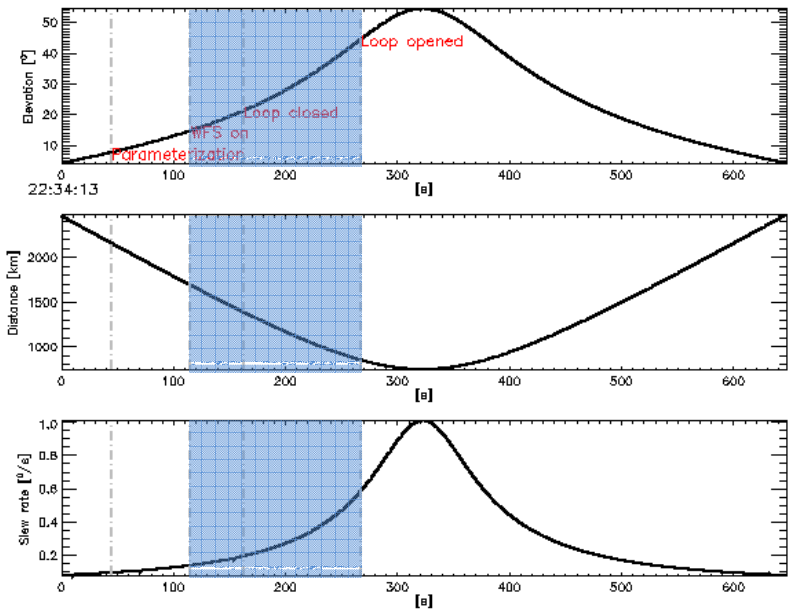

Fig. 2. Top: elevation as a function of time since the rise of the satellite Middle: distance to satellite, bottom: antenna slew rate. Blue: WFS data acquisition.

\section{RESULTS}

\section{A. Wavefront analysis: spatial characteristics}

The wavefront is spatially sampled by the WFS. The local slope in front of each subaperture is estimated from the position of the focal spot (see Fig. 3). The wavefront on the telescope aperture is then reconstructed by inverting a slope measurement model with a linear approach. When the AO loop is closed, pseudo-open loop slopes are computed from residual slopes and command applied to the deformable mirror.

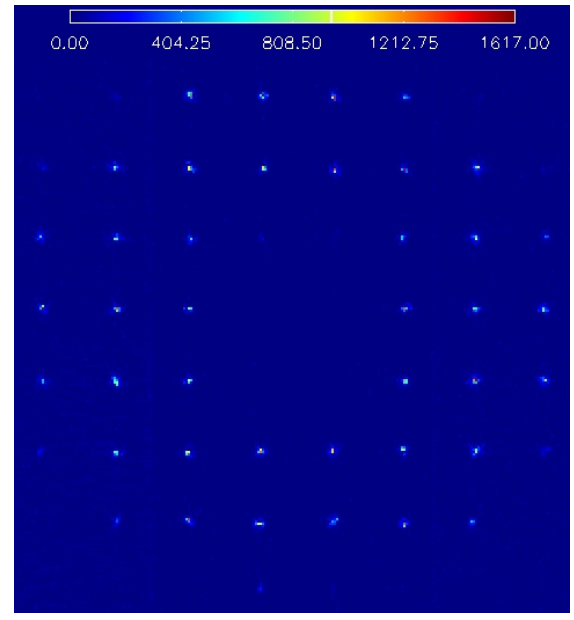

Fig. 3. Shack Hartmann focal plane

The set of data is decomposed into 35 data subsets evenly spaced with a period of $4 \mathrm{~s}$. Each set represents a $13.8 \mathrm{~s}$ duration. For each frame, the estimated wavefront is decomposed onto the first 45 Zernike modes (piston mode excluded). For each data subset, the variances per radial order are computed. They are reported on Fig. 4 (squares) for five elevations $\left(15.5^{\circ}, 19.8^{\circ}, 28.2^{\circ}, 32.6^{\circ}\right.$ and $\left.41.9^{\circ}\right)$.
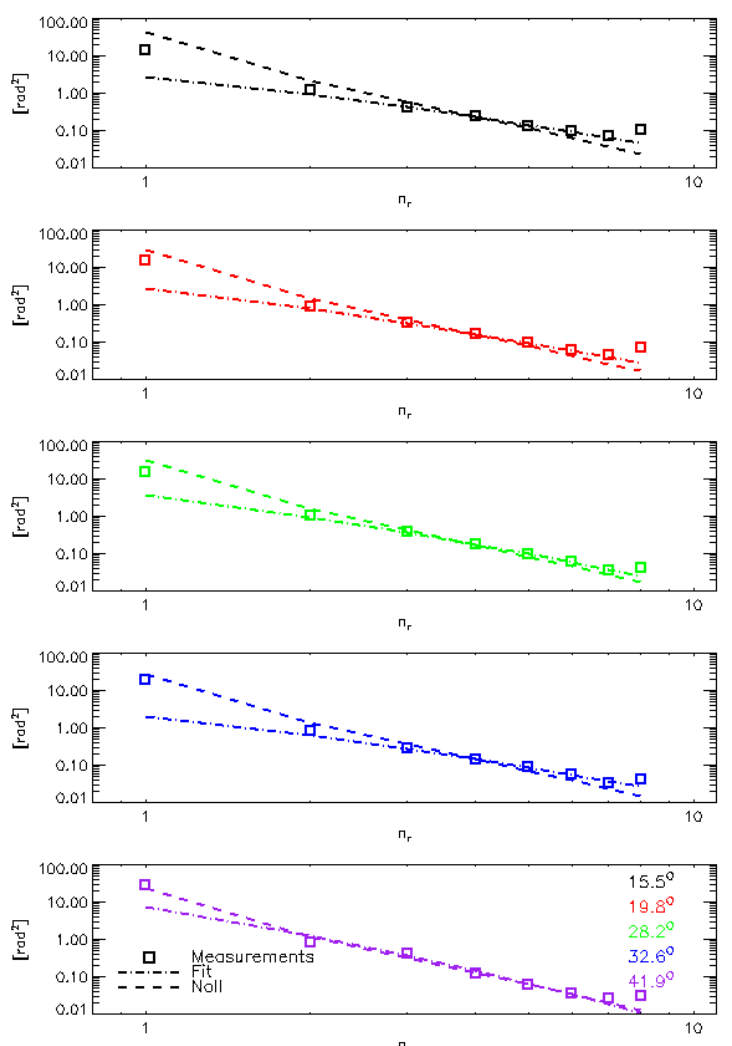

Fig. 4. Wavefront variance per radial order of Zernike polynomials for the five selected elevations (squares), $-11 / 3$ power law (dotted line)

An evaluation of the Fried parameter at $500 \mathrm{~nm}$ is performed from the radial variances given by Noll [8] (see Fig. 5). The radial orders 1 and 2 are not used in the $r_{0}$ estimation because they integrate the effect of the turbulence but also the contribution of vibrations, pointing error and outer scale, which could compromise the reliability of $\mathrm{r}_{0}$ estimation. To avoid the influence of aliasing, only radial orders up to six are considered. The estimation is performed for each radial degree as the radial variance may not follow the $\left(\mathrm{n}_{\mathrm{r}}+1\right)^{11 / 3}$ power law expected from Kolmogorov theory. The variances obtained by averaging the estimated $r_{0}$ and by assuming a $-11 / 3$ power law are plotted in dashed lines. The mixed line is the best fit of the Zernike variances with a power law, without constraining the power value. For radial orders greater than 2, the $-11 / 3$ power law is well satisfied except for $15.5^{\circ}$ elevation. At elevations lower than $20^{\circ}, r_{0}$ becomes significantly smaller than the subaperture diameter and as it is shown in Fig. 3 the focal spots of the SHWFS are enlarged since the phase fluctuations in the subapertures are no more negligible. In fact, the thresholded center of gravity of such spots introduces some white noise in the slope estimation which propagates in the phase reconstruction, hence degrading the Zernike coefficients of 
highest orders. The standard deviation on the estimation of $r_{0}$ is also reported in Fig. 5. It corresponds to the dispersion of the $\mathrm{r}_{0}$, estimated from each radial order (i.e. from 3 to 6 ).

Assuming the $\mathrm{C}_{\mathrm{n}}{ }^{2}$ vertical profile as unchanged in the surroundings of the telescope, the variation of $r_{0}$ as a function of elevation (see for example Roddier [9]) is given by:

$$
r_{0}(\theta)=(\sin \theta)^{3 / 5} r_{0}
$$

where $r_{0}$ is the Fried parameter at Zenith and $\theta$ is the elevation angle.

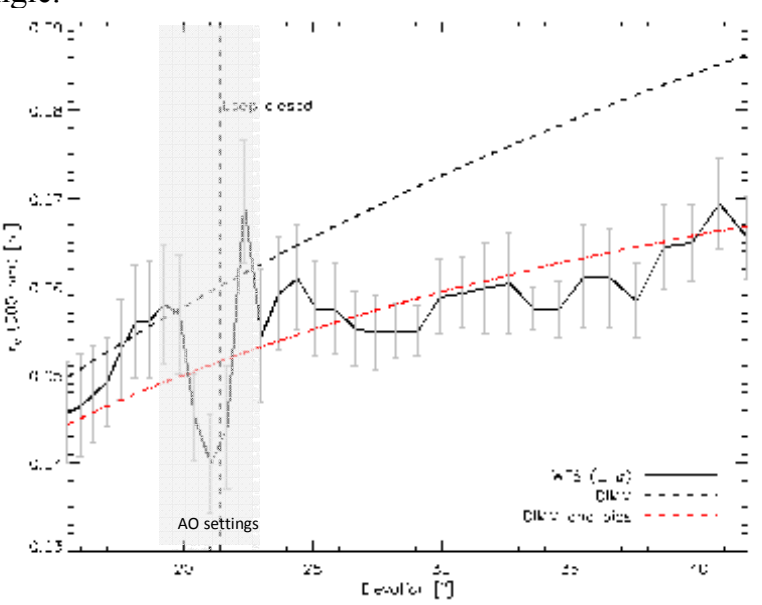

Fig. 5. Fried parameter evaluation as a function of elevation.
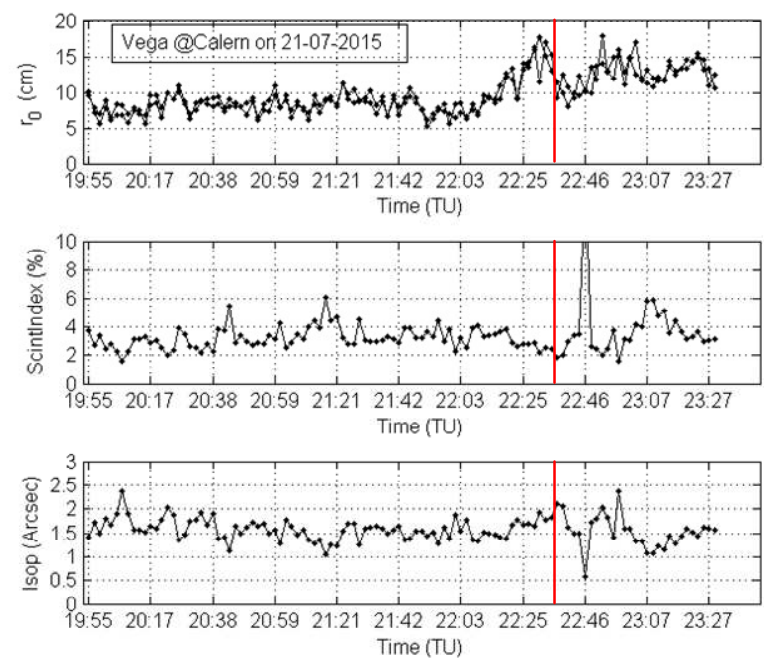

Fig. 6. Turbulence parameters measured with GDIMM on the $21^{\text {st }}$ of July. The vertical line indicates the time corresponding to $43^{\circ}$ elevation.

A GDIMM measurement is provided every 105 seconds during the pass. As illustrated by the plot, the value of $r_{0}(\theta)$ estimated with the beam coming from the satellite LOS does not correspond to the value of $r_{0}$ obtained from GDIMM measurement. The difference may be explained by a constant bias in phase variance. This bias corresponds to wavefront phase perturbations that do not depend on elevation, and that do not affect GDIMM measurements. Their origin may be due, for instance, to local turbulence effects related to the $1.5 \mathrm{~m}$ telescope itself (dome effect or temperature gradients close to the tube). In this case, the biased $r_{0}$ denoted $r_{0, \text { bias }}$ depends on the elevation as:

$$
\mathrm{r}_{0, \text { bias }}(\theta)=\left(\operatorname{bias}^{-5 / 3}+\mathrm{r}_{0}(\theta)^{-5 / 3}\right)^{-3 / 5}
$$

Based on this analysis, we obtain a mean value: bias $=12,6 \mathrm{~cm}$ from the experimental data. Once this bias taken into account, GDIMM measurements and WFS measurements have been compared, except around the time when the AO loop is closed. At this moment, the modifications of system parameters degrade the WFS data and may explain highly varying Fried parameters between $19^{\circ}$ and $23^{\circ}$. Further investigations are needed to assess the reliability of the estimation in this elevation range. Outside this time window, $r_{0}$ measurements from WFS and GDIMM are consistent considering that the distance between both instruments was $80 \mathrm{~m}$ and that both measurements was not perfectly simultaneous (the correlation time of $r_{0}$ measurements is estimated to $10 \mathrm{~min}$ from GDIMM measurements). Note that the wind at ground level was null according to the meteorological station of the observatory. Of course, additional similar measurements in various conditions would be of great interest to confirm this conclusion.

The phase variance estimated along the pass gives an idea of the fluctuations of the zenithal Fried parameter, as estimated with (1), during $210 \mathrm{~s}$, for an elevation angle from $15^{\circ}$ to $43^{\circ}$. It appears that the variability of the zenithal Fried parameter, estimated by the standard deviation of the normalized difference between the biased $\mathrm{r}_{0}$ from GDIMM and the measured $r_{0}$, does not exceed $10 \%$ on the whole trajectory.

\section{B. Wavefront analysis: temporal characteristics}

Temporal statistics of the measured wavefront is given here. At given elevation, the temporal power spectral densities (TPSD) of the Zernike coefficients of same radial orders are added. The result is plotted in Fig. 7 for radial orders from 2 to 6 . The shape of the TPSD per radial order gives an insight into the temporal correlations of the wavefront. These correlations may be related to the turbulence structure along the LOS owing to the apparent velocity of the satellite and assuming Taylor frozen flow hypothesis [10]. 


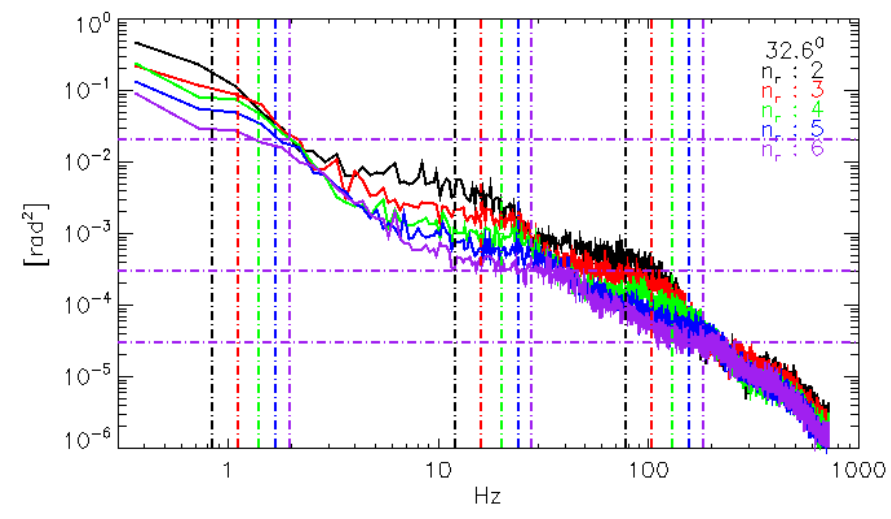

Fig. 7. Temporal spectra for radial orders from 2 to 6 for the $32.6^{\circ}$ elevation. Cutoff frequencies for each radial orders are reported (vertical mixed color lines).

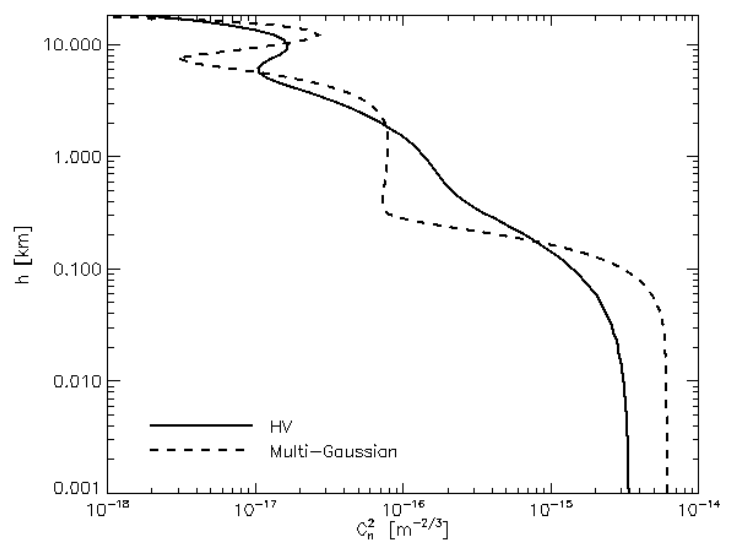

Fig. 8. Custom $\mathrm{C}_{\mathrm{n}}^{2}$ (Multi-Gaussian) and Hufnagel - Valley profile.

A cutoff frequency identified in the TPSD is related to the apparent velocity of a turbulent layer according to:

$$
v_{\mathrm{c}, 1}=0,3\left(\mathrm{n}_{\mathrm{r}}+1\right) \frac{\mathrm{V}_{\perp}}{\mathrm{D}}
$$

where $\mathrm{D}$ is the telescope diameter and $\mathrm{V}_{\perp}$ the norm of the apparent velocity of the layer. The analysis of the TPSDs enables to identify three major cutoff frequencies for the case $\mathrm{n}_{\mathrm{r}}=6: \mathrm{v}_{\mathrm{c}, 1}=1,5 \mathrm{~Hz}, \mathrm{v}_{\mathrm{c}, 2}=28 \mathrm{~Hz}, \mathrm{v}_{\mathrm{c}, 3}=190 \mathrm{~Hz}$, reported in the Fig. 7 (vertical plots in purple color lines) and hence three major layers,. The corresponding apparent velocities, obtained by inverting (3), are respectively, $\mathrm{V}_{\perp, 1}=1 \mathrm{~m} / \mathrm{s}, \mathrm{V}_{\perp, 2}=20 \mathrm{~m} / \mathrm{s}$, $\mathrm{V}_{\perp, 3}=133 \mathrm{~m} / \mathrm{s}$. The first cutoff frequency is slightly visible for radial order 6 . Its existence is consolidated by the analysis of the smaller radial orders where the cutoff frequencies computed from Eq. (3) and $\mathrm{V}_{\perp, 1}$ are reported (mixed color lines).

The major contributor to apparent velocity of the turbulent layers may be attributed to the satellite displacement. Natural wind is therefore neglected in the following. It is possible to link the apparent layer velocity with the layer distance from the telescope and therefore with the layer height: from basic trigonometric considerations, the altitude of the turbulent layer responsible for PSD cutoff frequency is approximated by:

$$
\mathrm{h}_{1}=\frac{\mathrm{V}_{\perp, 1}}{\omega} \sin \theta
$$

where $\omega$ is the angular velocity of the telescope. Hence, the obtained altitudes of the three turbulent layers are $h_{1}=100 \mathrm{~m}$, $\mathrm{h}_{2}=1.3 \mathrm{~km}$ and $\mathrm{h}_{3}=12 \mathrm{~km}$.

A multi-gaussian (MG) profile is built from this three layer decomposition in order to understand if a few layers only are sufficient to describe our results. The profile is composed of three Gaussian layers located at altitude $h_{k}$ of thickness $\sigma_{k}$ and turbulence strength $\mathrm{C}_{\mathrm{n}}^{2}\left(\mathrm{~h}_{\mathrm{k}}\right)$ :

$$
\mathrm{C}_{\mathrm{n}}^{2}(\mathrm{~h})=\sum_{\mathrm{k}=1}^{3} \mathrm{C}_{\mathrm{n}}^{2}\left(\mathrm{~h}_{\mathrm{k}}\right) \exp \left[\left(\frac{\mathrm{h}-\mathrm{h}_{\mathrm{k}}}{\sigma_{\mathrm{k}}}\right)^{2}\right]
$$

The height $\left(\mathrm{h}_{\mathrm{k}}\right)_{\mathrm{k}=[1,2,3]}=[0,1.3,12.4] \mathrm{km}$ are given by the TPSD analysis, $\left(\sigma_{\mathrm{k}}\right)_{\mathrm{k}=[1,2,3]}=[0.1,3,3] \mathrm{km}$ and $\left(\mathrm{C}_{\mathrm{n}}{ }^{2}\right)_{\mathrm{k}=[1,2,3]}=\left[6,110^{-15}\right.$, $\left.710^{-17}, 310^{-17}\right]$. The turbulence strength of high altitude layers and their thickness have been chosen to fit scintillation index temporal spectrum (see next paragraph for the results of the fit). The $\mathrm{C}_{\mathrm{n}}{ }^{2}$ profile is plotted in Fig. 8 and compared with a typical Hufnagel-Valley model. The $\mathrm{C}_{\mathrm{n}}{ }^{2}$ at ground level of $\mathrm{H}-\mathrm{V}$ model is adapted so that the two profiles present the same Fried parameter (here $10 \mathrm{~cm}$ along the LOS at $976 \mathrm{~nm}$ ). The MG model has been used as input of an analytical model of radial PSD to evaluate the pertinence of the analysis. The Zernike phase variance spectrum obtained from the MG model for radial orders 2 to 6 is plotted in Fig. 9, as well as the spectrum obtained from the data. The low frequencies are badly fitted due to the presence of slowly varying aberrations that are not taken into account in the model. For higher frequencies the model adequately fits the data.

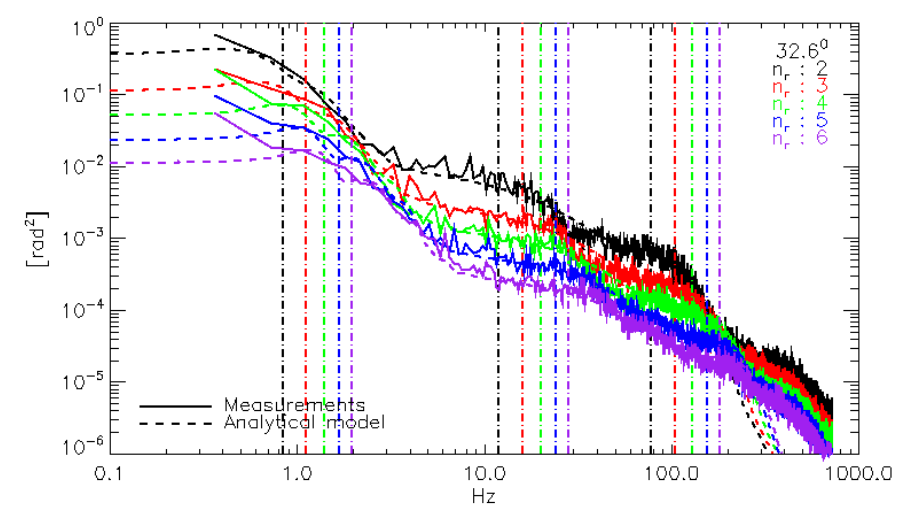

Fig. 9. Results of analytical model compared to temporal PSD for radial orders 2 to 6 for $32.6^{\circ}$ elevation.

The richness of wavefront data is illustrated here as they enable to estimate turbulence parameters with a rather straightforward approach. The same approach could be applied to the whole set of data to obtain an insight into the variability of turbulence profiles along the trajectory of the satellite. A preliminary trend 
can be spotted by studying the evolution of temporal power spectrum as a function of elevation. The measured TPSDs of Zernike coefficients of radial order 6 are plotted in Fig. 10 for increasing elevations. Between $28.2^{\circ}$ and $41.9^{\circ}$, the TPSDs present the same shape, the cutoff frequencies attributed to high altitude layers are similar. Even more, in this angular interval, turbulence distribution along the LOS seems to be very comparable. This interval corresponds to a $1.7 \mathrm{~km}$ footprint for a $10 \mathrm{~km}$ high altitude layer.

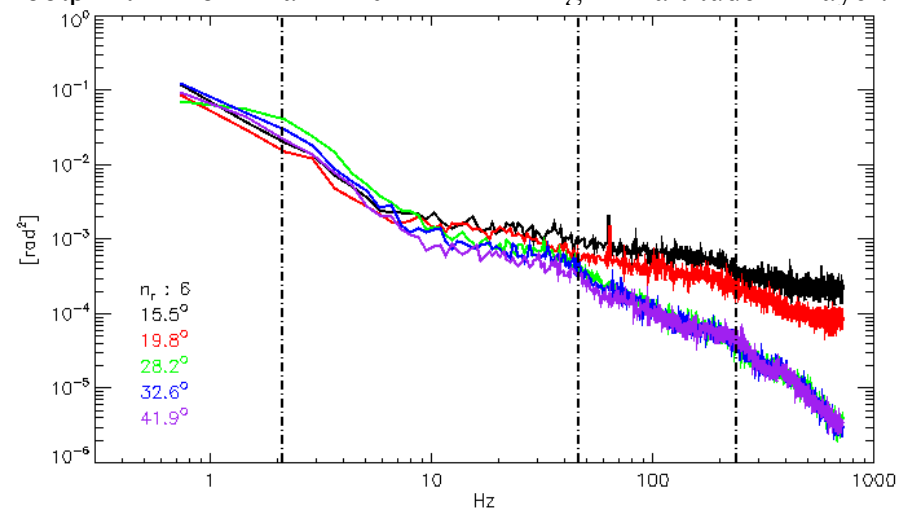

Fig. 10. Evolution of TPSD of sixth radial order as for increasing elevation.

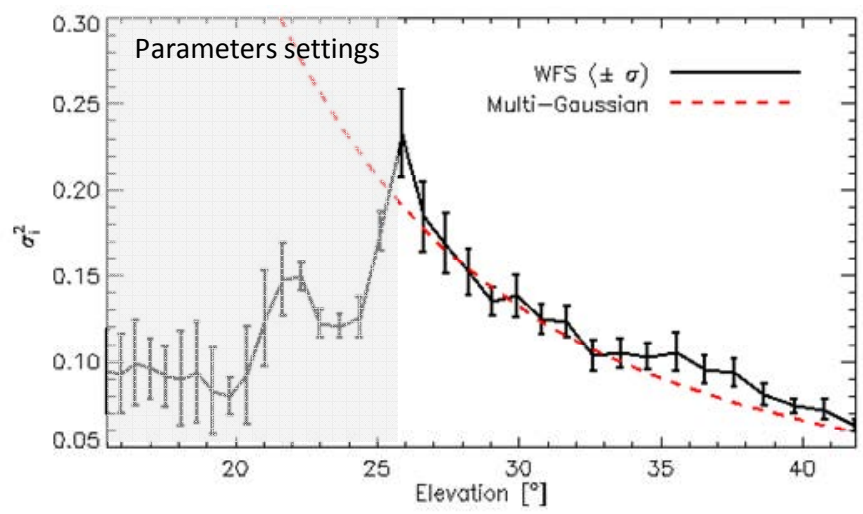

Fig. 11. Evolution of the scintillation index as a function of elevation from measurements (black), and from multi-gaussian profile (dashed red).

\section{WFS intensities data: scintillation index investigation}

A vector of intensities is provided by the WFS at every frame. For each data set, the normalized intensity fluctuations $i_{k, j}$ per subaperture are computed according to:

$$
i_{k, j}=\frac{I_{k}, j}{\frac{1}{N_{j}} \sum_{k=1}^{N_{j}} I_{k, j}}
$$

where the subscript $\mathrm{j}$ stands for the data set and $\mathrm{k}$ corresponds to the $\mathrm{k}^{\text {th }}$ measurement in the data-set. $\mathrm{N}_{\mathrm{j}}$ is the number of samples in each data set $\left(\mathrm{N}_{\mathrm{j}}=20\right.$ kilo-samples here $)$.

We have analyzed these data with regards to the scintillation index. This analysis has to be realized with caution. During the acquisition of the data set ( 13 seconds $)$ some of the acquisition parameters may vary. For instance, before pupil stabilization is turned on (when AO loop is closed), the position of the telescope aperture projected in the WFS lenslet array may be shifted up to a half of the size of a microlens. For this reason, only unocculted subapertures are exploited to compute the scintillation index. Moreover to overcome the fluctuations of the power delivered by the laser source or other sources of low frequencies fluctuations, the scintillation index is computed from the integral of the temporal power spectral density after the filtering of fluctuations below $30 \mathrm{~Hz}$. The evolution of the scintillation index measurement as a function of elevation is plotted in Fig. 11.

The results of scintillation index estimation from the Multi Gaussian $\mathrm{C}_{\mathrm{n}}{ }^{2}$ profile is also reported on the figure. Below $26^{\circ}$, acquisition parameters were modified to optimize the AO loop performances. For elevations above $26^{\circ}$, the parameters were set and no longer changed. The measured scintillation index decreases with the elevation as it is expected from theory. The MG profile correctly fits the measurements. For the $32.6^{\circ}$ elevation, this is expected as the profile parameters where adjusted to reproduce the scintillation index temporal spectra at this elevation. However, above $25^{\circ}$, the estimated parameters are sufficient to reproduce the evolution of the scintillation index as a function of the elevation on an elevation range greater than $15^{\circ}$ : the discrepancy between the scintillation index obtained from the data and the one obtained from the analytical model does not exceed $7 \%$. Below $25^{\circ}$, the scintillation index processed from the data needs to be consolidated due to the modifications of acquisition parameters.

\section{WFS intensities data: statistical characteristics}

We investigate here the statistical characteristics of intensities provided by WFS. The TPSD of the scintillation index computed according to (5) are plotted in Fig. 12 for the previously studied elevations, together with the results of the analytical model described in [11]. For elevations $28.2^{\circ}$ and above, the analytical model fits well the data. For elevation $19.8^{\circ}$ and below, this is not the case. No clear-cut conclusion can be drawn for this time period as it corresponds to several modifications of the acquisition parameters not all taken into account in the data reduction. For instance, current data reduction does not take into account threshold level influence on the intensity measurements whereas the portion of thresholded intensity might be significant. This is particularly true in open loop and at low elevations, as focal spots might be spread over several pixels. A more detailed post processing of the data at low elevation is needed to adjust the values. This will be done by using raw WFS images.

Once taken into account differences between GDIMM and WFS measurements (aperture diameter, wavelength, elevation and exposure time), it appears that scintillation index obtained from WFS data is larger than the scintillation index provided by GDIMM by approximately a factor four for elevations greater than $25^{\circ}$. This discrepancy between GDIMM measurements of scintillation index and the estimation from 
WFS needs to be investigated. The comparison of measurements performed simultaneously on the same source will certainly help to consolidate the cross-calibration of both instruments. Such correlated data were recently acquired and will be soon reduced.

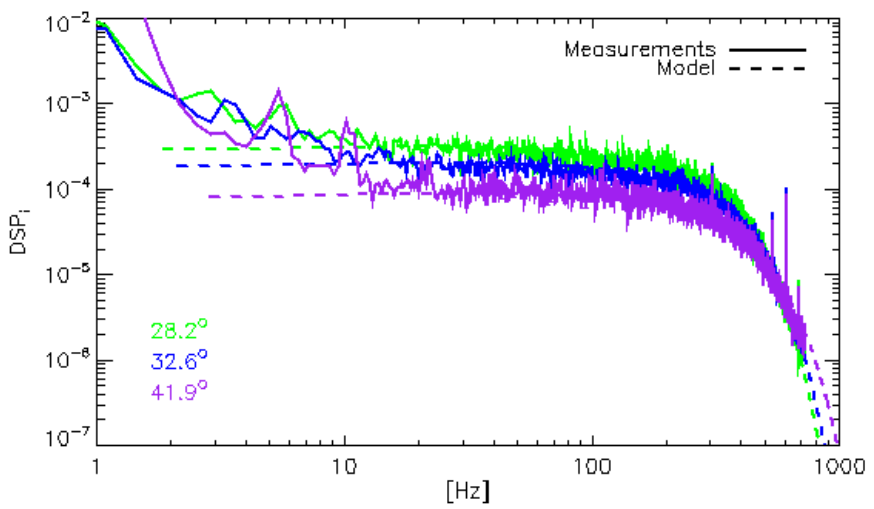

Fig. 12. Flux fluctuations power spectral densities for $15.5^{\circ}, 19.8^{\circ}, 28.2^{\circ}$, $32.6^{\circ}$ and 41.9 .

Intensities fluctuations histograms are plotted in Fig. 13 for the three elevations below $28.2^{\circ}$ together with a log-normal law which parameters (average and variance) are estimated from the data. The histograms show that the log-normal distribution assumed by small perturbation approximation is not satisfied. This is consistent with the Rytov log-amplitude variance of 0.3 computed from $\mathrm{MG} \mathrm{C}_{\mathrm{n}}{ }^{2}$ profile for $28,2^{\circ}$. The distance to the log-normal law decreases as elevation increases as it is expected, due to a shorter propagation path through the atmosphere.

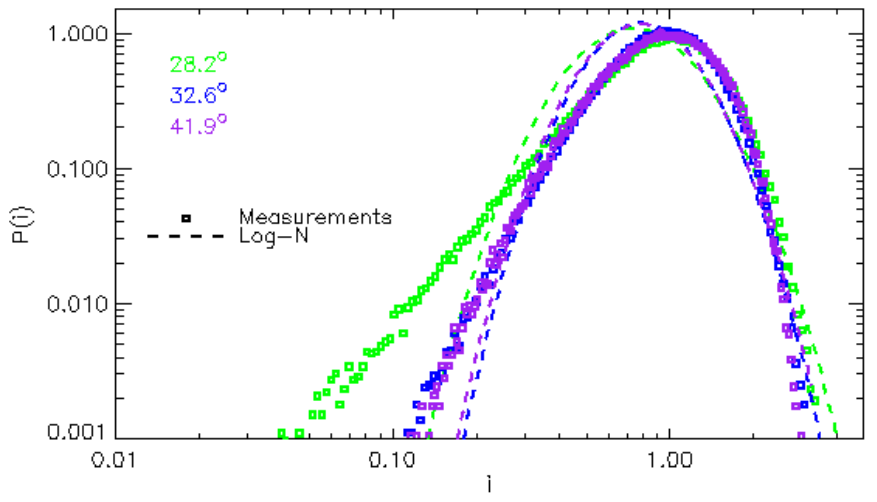

Fig. 13. Histograms of flux fluctuations and log-normal probability density function for the five selected elevations.

\section{CONCLUSION}

Wavefront sensor data (slopes and intensities) were acquired in July 2015 on an optical link provided by SOTA. During a single satellite pass, more than 2 minutes of data were collected between $15.5^{\circ}$ and $41^{\circ}$ of elevation. First results of data reduction, as well as a comparison to analytical models, have been presented. We show that the measured $\mathrm{r}_{0}$ may be described using a unique $\mathrm{C}_{\mathrm{n}}{ }^{2}$ vertical profile including a bias to account for the local turbulence close to the telescope. The data analysis shows that, during this nighttime experiment performed on an astronomical site, without any wind measured at ground level, the Fried parameter was very stable during the pass. The standard deviation of the difference between the $r_{0}$ given by the measurement and the $r_{0}$ given by the MG model was smaller than $10 \%$.

The temporal analysis of the recorded wavefronts revealed the presence of three main turbulence layers on the LOS. A multiGaussian $\mathrm{C}_{\mathrm{n}}{ }^{2}$ profile has been proposed and parameterized with the measured data. It correctly accounts for wavefront temporal characteristics at the considered elevation. The Multi-Gaussian profile is also well adapted to fit the scintillation index evolution for elevations greater than $25^{\circ}$. As for Fried parameter, the scintillation index shows a striking stationarity as its standard deviation is below $7 \%$ on a $15^{\circ}$ range. For a turbulent layer located at a $10 \mathrm{~km}$ altitude, this corresponds to a typical correlation scale greater than $2.5 \mathrm{~km}$. Data recorded simultaneously by GDIMM and WFS will be exploited to cross-check the figures. Further investigations are required to consolidate the conclusions for elevations below $25^{\circ}$. Wave optics simulations will be performed to investigate the effect of strong turbulence on the instruments.

\section{ACKNOWLEDGMENT}

The author thanks the whole staff of Côte d'Azur Observatory for its kindness and availability during the experiments.

\section{REFERENCES}

[1] Védrenne, N. et al., Turbulence effects on bi-directional ground-tosatellite laser communication systems, "Conference on Space Optical Systems and Applications", ICSOS, France, October 9-12, 2012.

[2] Védrenne, N. et al., "Characterization of Atmospheric Turbulence for LEO to Ground Laser Beam Propagation at Low Elevation Angles", ICSOS, Japan, May 7-9, 2014.

[3] Koyama, Y., Takayama, T., Akioka, M.,Takenaka, H., Munemasa, Y., Toyoshima, M., "Completion of the small optical transponder for salellite-ground laser communication demonstration", 19th conference on $\mathrm{Ka}$ band and broadband communications, Florence, Italy, October 14-17, 2013.

[4] cwe.ccsds.org/sls/docs/SLS-OPT/Meeting Materials/2014/Fall 2014 London meeting/Proposal of physical layer elements for a CCSDS OPT standard suitable for LEO to ground High Data Rate DTE link----.pdf

[5] E. Samain, A. Abchiche, D. Albanese, et al. "MeO: The new French lunar laser station. In Proceedings of the 16th International Workshop on Laser Ranging", October, 2008.

[6] Aristidi, E., Fanteï-Caujolle, Y., Ziad, A., Dimur, C., Chabé J. and Roland, B., "A new generalized differential image motion monitor", SPIE-Proceedings 9145, Canada, July 22, 2014.

[7] Petit, C., Védrenne, N., Michau, V., Samain, E., Toyoshima, M., Akioka, M., Kolev, D., Munemasa, Y., Takenaka, H., Iwakiri,V., Artaud, G., Issler, J.-L., "Adaptive Optics Results with SOTA", ICSOS, USA, October 27-28, 2015.

[8] Noll, R., "Zernike polynomials and atmospheric turbulence," J. Opt. Soc. Am. 66, 207-211, 1976. 
[9] Roddier, F., "The Effects of Atmospheric Turbulence in Optical Astronomy", In: E. Wolf, Editor(s), Progress in Optics, Elsevier, 1981, Volume 19, Pages 281-376.

[10] Conan, J.-M., Rousset, G., Madec, P.-Y. "Wave-front temporal spectra in high-resolution imaging through turbulence," J. Opt. Soc. Am. A 12, 1559-1570, (1995).
[11] Robert, C., Conan, J.-M., Michau, V., Renard, J., Dalaudier,F., "Retrieving parameters of the anisotropic refractive index fluctuations spectrum in the stratosphere from balloon-borne observations of stellar scintillation," J. Opt. Soc. Am. A 25, 379-393 (2008). 